\title{
Hepatocellular Carcinoma in Micronesians, a Growing Pacific Islander Population in the U.S.
}

\author{
Eric M. Wu1 ${ }^{1}$ Brenda Y. Hernandez ${ }^{2}$, Linda L. Wong ${ }^{1 *}$ \\ ${ }^{1}$ Department of Surgery, University of Hawaii, John A. Burns School of Medicine, Honolulu, USA \\ ${ }^{2}$ Cancer Center, University of Hawaii, Honolulu, USA \\ Email: *hepatoma@aol.com
}

How to cite this paper: Wu, E.M., Hernandez, B.Y. and Wong, L.L. (2018) Hepatocellular Carcinoma in Micronesians, a Growing Pacific Islander Population in the U.S. Open Journal of Gastroenterology, 8, 223-233.

https://doi.org/10.4236/ojgas.2018.86025

Received: May 21, 2018

Accepted: June 26, 2018

Published: June 29, 2018

Copyright $\odot 2018$ by authors and Scientific Research Publishing Inc. This work is licensed under the Creative Commons Attribution International License (CC BY 4.0).

http://creativecommons.org/licenses/by/4.0/

\begin{abstract}
Background: Micronesians are a rapidly growing population in Hawaii and the continental U.S. Hepatitis B is prevalent in Micronesia, but the consequences in those migrating to the U.S. are unclear. This study characterizes hepatocellular carcinoma in Micronesians with comparisons to other Pacific Islanders. Methods: A retrospective evaluation was undertaken of 40 Micronesian and 142 Non-Micronesian Pacific hepatocellular carcinoma patients diagnosed in a large clinical practice in Hawaii from 1993-2017. Results: All Micronesians were born outside of the U.S. Micronesians were younger at diagnosis ( 52.0 vs. 60.2 years), had higher rates of hepatitis B ( $80 \%$ vs. $31.2 \%$ ), a family history of hepatocellular carcinoma (17.5\% vs. $4.4 \%)$ and more tumors $<5 \mathrm{~cm}$ (64.1\% vs. $45.2 \%)$. Non Micronesian Pacific Islanders had higher rates of Hepatitis C ( $48.6 \%$ vs. $22.5 \%)$ and hypertension ( $61.7 \%$ vs. $32.4 \%)$. There was no difference in the proportions of screen-detected tumors, those meeting Milan criteria, tumor stage, or liver function. Micronesians had better 5-year survival rates ( $48 \%$ vs. $16.7 \%$ ). Conclusion: Hepatocellular carcinoma in Micronesians is primarily Hepatitis B-related. Micronesians were equally likely to have hepatocellular carcinoma found with screening, undergo transplant and had better survival. Our study emphasizes the need for hepatitis B screening and hepatocellular carcinoma surveillance in Micronesians.
\end{abstract}

\section{Keywords}

Hepatocellular Carcinoma, Micronesians, Hepatitis B, Screening

\section{Introduction}

Hepatocellular Carcinoma (HCC) is currently the fifth leading cause of cancer death in the United States, with an estimated incidence of 8.6 per 100,000 that 
continues to rise by approximately $2.7 \%$ annually [1]. HCC is more common in men, and is seen most often in the Asian/Pacific Islander and American Indian/Alaskan Native populations. The most common risk factors for HCC include underlying chronic hepatitis B and C infections ( $~ 80 \%$ of cases), cirrhosis of any cause and other chronic liver disease [2]. From 2010-2014, Hawaii had the highest incidence rate of HCC (11.29/100,000 people) [1], which is likely due to the large Asian/Pacific Islander population and continuous immigration from Asia and the Pacific Basin. Given these conditions, Hawaii is an ideal state to examine HCC among different Pacific Islander subpopulations.

Micronesians are among the newest and fastest growing groups of migrants to the U.S. They hail from Micronesia, a region in the western Pacific comprised of several nations (Federated States of Micronesia, Republic of Marshall Islands, Republic of Kiribati, Republic of Palau, Republic of Nauru) and U.S. territories including the Commonwealth of the Northern Mariana Islands, Guam and Wake Island. The U.S. Government Accountability Office estimates that about 56,000 Micronesians reside in the U.S. [3], with an estimated 15,000 Micronesians now living in Hawaii. Although they comprise only 1\% of Hawaii's population [4], annual healthcare expenditure in 2007 on Micronesians was estimated at over $\$ 37$ million [5]. Emigration from Micronesia is driven by multiple factors including decreased U.S. funding, scarce employment opportunities, natural disasters, and a lack of health infrastructure. The Compact of Free Association (COFA) agreement between the U.S. and the Free Associated States (including the Federated States of Micronesia, Marshall Islands, and Palau) allows citizens to live, work and study in the U.S. permanently without a visa, further encouraging migration. In a survey of Micronesian migrants, Hezel et al. found that among those migrating to Hawaii, $11 \%$ moved specifically for medical reasons, more so than any other site of immigration [6]. The health status of Micronesians is complex and characterized by both non-communicable diseases as seen in the developed nations such as obesity, diabetes mellitus type 2, hypertension and heart disease, and significant infectious disease burden [7]. For HCC, Palafox et al. identified hepatitis B and alcohol abuse as major risk factors in Micronesians [8].

Micronesian migration to Hawaii continues to increase and the health services utilization by this population is significant [6]. While infectious diseases including hepatitis B have been studied in this population, there have been no studies characterizing HCC in Micronesians. We aim to describe the disease course in these patients from risk factors to survival, and then compare these factors with a group of Non-Micronesian Pacific Islanders (NMPI).

\section{Methods}

\subsection{Study Participants}

This is a retrospective study of a prospectively collected database of patients with HCC. A total of 1206 patients were referred to a group of surgeons affiliated with 
the St. Francis Medical Center (1993-2012), and at the Queen's Medical Center (1993-2016). These medical centers had the only clinic dedicated to liver diseases, the only liver transplantation program in the state, and is the only referral center for liver disease for the U.S. territories in the Pacific Basin (including American Samoa, Guam, Commonwealth of the Northern Mariana Islands, and the Republic of the Marshall Islands). From the database, a total of 182 patients self-identified as Pacific Islander (including any patient with $50 \%$ or higher of any Pacific Islander ethnicity), and 40 of these patients self-identified as Micronesian (including Chuukese, Guamanian, Marshallese, Palauan, and Yapese). The remaining 142 NMPI were mostly comprised of Hawaiians, Samoans, and Tongans. These two populations were analyzed and compared to each other. This study was approved as exempt from review by the University of Hawaii Human Studies Program.

\subsection{Data Collection}

HCC was diagnosed histologically by percutaneous biopsy or at surgery. In the first decade and consistent with the previous United Network for Organ Sharing policy regarding transplant for HCC, patients without histologic confirmation were included if they had a history of chronic liver disease and a mass at least 2 $\mathrm{cm}$ in size seen on 2 imaging studies (ultrasound, computed tomographic [CT] scan, or MRI) and one of the following: vascular blush seen on CT scan or MRI, alpha-fetoprotein (AFP) greater than $200 \mathrm{ng} / \mathrm{mL}$, or arteriogram confirming the tumor. More recently, the diagnosis of HCC was made with only imaging if a contrast-enhanced study (dynamic CT or MRI) showed typical arterial enhancement with "washout" in the venous phase as described by the American Association for the Study of Liver Disease guidelines.

Demographic data including age, sex, birthplace, insurance, and self-reported ethnicity were collected through patient interviews. Ethnicity was categorized as "White," "Asian", or "Pacific Islander". Patients that self-identified as Micronesian were also asked to identify their specific origin from within Micronesia. Data collected on medical history included diabetes mellitus, hypertension, hyperlipidemia, obesity (BMI > 30), other cancers, smoking, and family history of cancer. Risk factors for HCC including viral hepatitis, alcohol abuse $(>2$ drinks/day for at least 10 years), and other chronic liver diseases were identified. Screenable disease was defined as having hepatitis B, C, or alcohol abuse.

Laboratory data collected included bilirubin, albumin, prothrombin time, INR, creatinine, alanine aminotransferase (ALT), aspartate aminotransferase (AST), platelet count, and AFP. Model for End-Stage Liver Disease (MELD) score and Barcelona Clinic Liver Cancer (BCLC) score were also calculated. Tumor characteristics (size, number and location) and nodal status were identified and used to determine the AJCC stage. The presence of ascites and encephalopathy were identified and used to calculate Childs-Turcotte-Pugh (CTP) scores. These scores were expressed as Class A (CTP 5-6), B (CTP 7-9) or C 
(CTP > 9). Liver resection was considered in Child's A patients or early Child's B (CTP score of 7) patients without any evidence of ascites or encephalopathy. A liver transplant was considered in patients who were unresectable for technical reasons or had decompensated liver disease, but were stage T1 or T2 (Milan criteria).

Most patients awaiting transplantation received percutaneous radiofrequency ablation (RFA) or transarterial chemoembolization (TACE). For purposes of analysis, liver resection and transplantation were considered to be curative forms of treatment. Locoregional therapy was defined as including RFA, TACE, embolization, cryoablation, or yttrium-90. The proportion of patients with HCC detected by screening was noted. Although our Liver Center recommends that community physicians screen viral hepatitis and chronic liver disease patients with AFP and liver ultrasound every six months, there was no standard screening protocol used in this cohort. Referring physicians used a combination of AFP and imaging (ultrasound, CT, or MRI) at variable intervals. We defined HCC as detected by screening if the referring physician stated that screening was done and the patient had a previous imaging study from 3 to 12 months prior. HCC not found on screening was either found with symptoms or asymptomatically with imaging done for unrelated reasons.

\subsection{Statistical Analysis}

All analyses were performed using Graphpad Prism (GraphPad Prism version 7.00 for Mac, GraphPad Software, La Jolla California USA, www.graphpad.com). Categorical variables were analyzed using Fisher's test and numerical variables were analyzed using unpaired two-tailed t-tests. Survival time was calculated as time from HCC diagnosis to death from all causes of mortality. The Kaplan-Meier method was used to calculate median survival and overall survival for both populations. $\mathrm{P}$ values $<0.05$ were considered significant.

\section{Results}

We analyzed 182 HCC patients that self-identified as either Micronesian or $\mathrm{Pa}$ cific Islander. There were 144 men (79.1\%) and 38 women with a mean age of 58 years. The Micronesian cohort consisted of 40 patients, with the remaining 142 classified as NMPI. In the overall cohort, hepatitis B and hepatitis C infection were seen in $42 \%$ and $42.9 \%$, respectively. Other risk factors included alcohol abuse (59.9\%), smoking (68.8\%), hypertension (54.6\%), diabetes (31.3\%), cirrhosis $(71.2 \%)$ and viral transmission risk factors (50.5\%). HCC presented symptomatically in $39 \%$ of patients and was screenable in $81.2 \%$ of cases. Curative treatment was performed on $26.8 \%$ of patients, with $32.4 \%$ not undergoing any treatment. Milan criteria were met in $41.8 \%$ of cases.

The Micronesian cohort was $85 \%$ male (34/40) and with a mean age of 52 years. All were born in Micronesia, with 20 (60.6\%) completing high school, and 10 (25\%) having private insurance. Nearly all had a screenable disease (97.5\%), 
but HCC was found with screening in 17.5\%. Smoking and alcohol history were present in $57.5 \%$ and $52.5 \%$, respectively. Most of the patients were hepatitis B positive ( $80 \%$ ), while only $22.5 \%$ were hepatitis C positive. Other metabolic risk factors included diabetes (22.5\%), hypertension (32.4\%), and hyperlipidemia (12.8\%), with a mean BMI of 30.9 and obesity in eighteen patients (57.4\%). $17.5 \%$ had a family history of HCC. For tumor and liver characteristics, $62.5 \%$ presented with a single tumor. $64.1 \%$ presented with a tumor less than $5 \mathrm{~cm}$. Thirty patients (75\%) were classified as AJCC stage I or II, $60 \%$ were Childs class $\mathrm{A}$, and $55 \%$ met Milan criteria. Cirrhosis was present in $82.5 \%$ with a mean MELD score of 12. For treatment, eight patients (20\%) received curative treatment, with four receiving resection (10\%) and four receiving transplant (10\%). Fifteen patients received locoregional therapy (37.5\%), and eighteen (45\%) received no treatment. For survival, $84 \%, 71.4 \%$ and $48 \%$ were alive at 1,2 and 5 years.

The NMPI cohort was $77.5 \%$ male (110/142), with a mean age of 60.2 years. $81.5 \%$ were born in the US. $70.6 \%$ completed high school, and $31.7 \%$ had private insurance. $76.6 \%$ had a screenable disease, and $17.6 \%$ had HCC found with screening. Smoking and alcohol history were present in $72 \%$ and $62 \%$, respectively. $31.2 \%$ of patients had hepatitis B, while $48.6 \%$ had hepatitis C. Metabolic risk factors such as diabetes, hypertension, and obesity were present in $33.8 \%$, $61.7 \%$ and $48 \%$, respectively. Family history of HCC was present in $4.4 \%$. Regarding tumor characteristics, $66.2 \%$ presented with a single tumor. $45.2 \%$ presented with a tumor size $<5 \mathrm{~cm}$. $65.5 \%$ were classified as AJCC stage I or II, $57.7 \%$ of patients were classified as Childs Class A, and $38 \%$ met Milan criteria. Cirrhosis was present in $67.9 \%$, with a mean MELD score of 12 . For treatment $19 \%$ received curative treatment, with $15.5 \%$ receiving a liver resection and 3.5\% receiving liver transplant. $50.7 \%$ received some form of locoregional therapy, and $28.9 \%$ received no treatment. Survival at 1,2 and 5 years was $54.1 \%, 35.7 \%$ and $16.7 \%$.

Table 1 compares demographics and risk factors between the two populations. The NMPI had a significantly older population with higher rates of HTN and hepatitis $\mathrm{C}$, while Micronesians had significantly higher rates of hepatitis $\mathrm{B}$ and a family history of HCC. There was a significant difference in birthplace, with none of the Micronesians being born in the U.S. There were no significant differences in gender, educational attainment, having private insurance, smoking, alcohol abuse, DM, hyperlipidemia, or obesity.

In Table 2, tumor characteristics were compared between Micronesians and NMPI. Micronesians presented with smaller tumor sizes. There were no significant differences in tumor staging, HCC found with screening, ruptured HCC, early vs. late Child's Class, vascular invasion, meeting Milan criteria, presence of cirrhosis, or MELD score. Laboratory studies (Table 3) did not differ between the two populations.

The treatment of HCC (Table 4) did not significantly differ between the 
Table 1. Demographics and risk factors.

\begin{tabular}{cccc}
\hline & \multicolumn{3}{c}{ Comparison between Micronesians and } \\
Non-Micronesian PI \\
\cline { 2 - 4 } Characteristics & $\begin{array}{c}\text { Micronesian } \\
(\mathrm{n}=40)\end{array}$ & $\begin{array}{c}\text { Non-Micronesian } \\
\text { PI (n = 142) }\end{array}$ & P-value \\
\hline Mean age (SD) & 52 years (10.41) & 60.2 years (11.11) & $<0.0001$ \\
Male gender (\%) & $34 / 40(85)$ & $110 / 142(77.5)$ & 0.38 \\
Born in US (excluding territories) (\%) & 0 & $110 / 135(81.5)$ & $<0.0001$ \\
Finished high school (\%) & $20 / 33(60.6)$ & $72 / 102(70.6)$ & 0.29 \\
Private Insurance (\%) & $10 / 40(25)$ & $44 / 139(31.7)$ & 0.56 \\
HCC found with screening (\%) & $7 / 40(17.5)$ & $25 / 142(17.6)$ & 1 \\
\% with screenable disease & $39 / 40(97.5)$ & $108 / 141(76.6)$ & 0.002 \\
\% screened if + screenable disease & $7 / 39(17.9)$ & $25 / 108(23.1)$ & 0.65 \\
Smoking (\%) & $23 / 40(57.5)$ & $98 / 136(72.0)$ & 0.09 \\
Alcohol history (\%) & $21 / 40(52.5)$ & $88 / 142(62.0)$ & 0.36 \\
Hepatitis B (\%) & $32 / 40(80)$ & $44 / 141(31.2)$ & $<0.0001$ \\
Hepatitis C (\%) & $9 / 40(22.5)$ & $69 / 142(48.6)$ & 0.004 \\
Diabetes Mellitus (\%) & $9 / 40(22.5)$ & $48 / 142(33.8)$ & 0.25 \\
Family History of HCC (\%) & $7 / 40(17.5)$ & $6 / 136(4.4)$ & 0.011 \\
Hypertension (\%) & $12 / 37(32.4)$ & $71 / 115(61.7)$ & 0.002 \\
Hyperlipidemia (\%) & $5 / 39(12.8)$ & $31 / 135(23.0)$ & 0.19 \\
Mean BMI (SD) & $30.9(6.19)$ & $30.7(6.56)$ & 0.84 \\
BMI > 30 (\%) & $18 / 35(57.4)$ & $60 / 125(48.0)$ & 0.85 \\
\hline
\end{tabular}

BMI, Body Mass Index.

Table 2. Tumor and Liver characteristics.

\begin{tabular}{cccc}
\hline & \multicolumn{3}{c}{ Comparison between Micronesians and } \\
Non-Micronesian PI \\
\cline { 2 - 4 } Characteristics & $\begin{array}{c}\text { Micronesian } \\
(\mathrm{n}=40)\end{array}$ & $\begin{array}{c}\text { Non-Micronesian } \\
\text { PI (n = 142) }\end{array}$ & P-value \\
\hline Mean tumor size (cm) (SD) & $6.63(5.8)$ & $6.96(4.8)$ & 0.81 \\
Tumor size < 5 cm (\%) & $25 / 39(64.1)$ & $61 / 135(45.2)$ & 0.046 \\
Early stage I or II (\%) & $30 / 40(75)$ & $93 / 142(65.5)$ & 0.34 \\
Tumor > 10 cm (\%) & $10 / 39(25.6)$ & $28 / 135(20.7)$ & 0.52 \\
Ruptured HCC (\%) & $0 / 40(0)$ & $8 / 142(5.5)$ & 0.20 \\
Childs Class A (\%) & $24 / 40(60)$ & $79 / 137(57.7)$ & 0.85 \\
Vascular Invasion (\%) & $6 / 40(15)$ & $15 / 142(10.6)$ & 0.41 \\
Met Milan Criteria (\%) & $22 / 40(55)$ & $54 / 142(38.0)$ & 0.07 \\
BCLC (0 vs A, B, C, D) (\%) & $0 / 40(0)$ & $3 / 139(2.2)$ & $>0.9999$ \\
Cirrhosis (\%) & $33 / 40(82.5)$ & $93 / 137(67.9)$ & 0.08 \\
Ascites (\%) & $7 / 40(17.5)$ & $29 / 112(25.9)$ & 0.82 \\
Encephalopathy (\%) & $5 / 40(12.5)$ & $16 / 141(11.3)$ & 0.78 \\
Mean MELD (SD) & $12(6.20)$ & $12(5.73)$ & 0.87 \\
\hline
\end{tabular}

BCLC, Barcelona Clinic Liver Cancer staging system; MELD, Model for End-Stage Liver Disease. 
Table 3. Laboratory studies.

\begin{tabular}{|c|c|c|c|}
\hline \multirow{2}{*}{ Characteristics } & \multicolumn{3}{|c|}{$\begin{array}{l}\text { Comparison between Micronesians and } \\
\text { Non-Micronesian PI }\end{array}$} \\
\hline & $\begin{array}{l}\text { Micronesian } \\
\quad(\mathrm{n}=40)\end{array}$ & $\begin{array}{l}\text { Non-Micronesian } \\
\text { PI }(\mathrm{n}=142)\end{array}$ & P-value \\
\hline Mean AFP (SD) & $24535(68518)$ & $22679(74312)$ & 0.89 \\
\hline Normal AFP (<20 ng/dL) (\%) & $13 / 40(32.5)$ & $50 / 141(35.5)$ & 0.85 \\
\hline Mean bilirubin (SD) & $2.6(4.9)$ & $2.4(4.3)$ & 0.84 \\
\hline Mean albumin (SD) & $3.2(0.75)$ & $3.4(0.66)$ & 0.19 \\
\hline Mean prothrombin time (SD) & $15.1(2.6)$ & $14.7(2.1)$ & 0.28 \\
\hline Mean INR (SD) & $1.2(0.28)$ & $1.2(0.21)$ & 0.25 \\
\hline Mean platelet $\left(10^{3} / \mathrm{cc}\right)(\mathrm{SD})$ & $180.2(122.9)$ & $184.3(92.4)$ & 0.82 \\
\hline Mean creatinine (SD) & $1.26(1.23)$ & $1.1(0.74)$ & 0.39 \\
\hline Mean AST (SD) & $108(165.8)$ & $100.3(84.8)$ & 0.69 \\
\hline Mean ALT (SD) & $60(39.7)$ & $73.9(63.9)$ & 0.22 \\
\hline Mean Cholesterol (SD) & $188.5(70.3)$ & $174.4(57.3)$ & 0.50 \\
\hline Mean Triglycerides (SD) & $89.8(35.4)$ & $123.1(57.5)$ & 0.07 \\
\hline FIB4 (SD) & $5.3(4.1)$ & $5.0(3.9)$ & 0.67 \\
\hline APRI (SD) & $0.8(0.76)$ & $0.7(0.77)$ & 0.67 \\
\hline
\end{tabular}

AFP, Alpha-fetoprotein; INR, International Normalized Ratio; AST, Aspartate Aminotransferase; ALT, Alanine Aminotransferase; FIB4, Fibrosis-4; APRI, AST to Platelet Ratio Index.

Table 4. Initial treatment of hepatocellular carcinoma and outcomes.

\begin{tabular}{cccc}
\hline \multirow{2}{*}{ Characteristics } & \multicolumn{3}{c}{ Comparison between Micronesians and } \\
\cline { 2 - 4 } & $\begin{array}{c}\text { Micronesian } \\
(\mathrm{n}=40)\end{array}$ & $\begin{array}{c}\text { Non-Micronesian PI } \\
\mathrm{n} \mathrm{PI}(\mathrm{n}=142)\end{array}$ & P-value \\
\hline Curative treatment (Resection or Transplant) (\%) & $8 / 40(20)$ & $27 / 142(19.0)$ & 1 \\
Liver resection (\%) & $4 / 40(10)$ & $22 / 142(15.5)$ & 0.45 \\
Liver transplant (\%) & $4 / 40(10)$ & $5 / 142(3.5)$ & 0.11 \\
Locoregional therapy (\%) & $15 / 40(37.5)$ & $72 / 142(50.7)$ & 0.16 \\
No treatment (\%) & $18 / 40(45)$ & $41 / 142(28.9)$ & 0.08 \\
1-year survival (\%) & $32 / 38(84)$ & $72 / 133(54.1)$ & 0.0007 \\
2-year survival (\%) & $25 / 35(71.4)$ & $45 / 126(35.7)$ & 0.0002 \\
5-year survival (\%) & $12 / 25(48)$ & $18 / 108(16.7)$ & 0.0024 \\
\hline
\end{tabular}

populations with regards to liver transplantation, resection, curative treatment or locoregional therapy. Micronesians had significantly increased survival at 1,2 and 5 years. There was a median survival in Non-Micronesians of 502 days, and an undefined median survival in Micronesians ( $>50 \%$ alive at end of study).

\section{Discussion}

The HCC in Micronesians was predominantly hepatitis B related. This was likely vertically transmitted, as there was also a significantly higher family history of HCC. While children in the U.S. have routinely received the hepatitis B vaccina- 
tion for several decades, vaccination in some Asian and Pacific Island countries has not been uniformly practiced. Prior to the implementation of hepatitis B vaccination between 1986-1989, more than $70 \%$ of children on the Micronesian island of Chuuk were infected by ages 8 - 9 [9]. Despite efforts to more consistently vaccinate Micronesian children, completion of the vaccine series is still a challenge. As a result, approximately $15 \%$ of Micronesians are now chronically infected with hepatitis B and this is the primary risk factor for HCC [10]. Hopefully greater efforts at vaccination can reduce the incidence of HCC in the next generation of Micronesians, but it may take several decades to fully realize this benefit.

In the past, the primary risk factors for HCC were viral hepatitis and alcoholic cirrhosis, however the increasing incidence of HCC despite better control of hepatitis $\mathrm{B}$ and $\mathrm{C}$ may be attributed to the effect of metabolic factors, fatty liver disease and non-alcoholic steatohepatitis [11]. In this study, metabolic risk factors did not differ between Micronesians and NMPI, however obesity was present in about half of both groups. In comparison, the Centers for Disease Control (CDC) estimated the prevalence of obesity in the U.S. (2011-2014) at $36 \%$ [12]. Diabetes was present in $22 \%$ of Micronesians and $38 \%$ of NMPI; while not different between the groups, the prevalence is higher than the general population (9.4\%) and all Native Hawaiians/Pacific Islanders (15.2\%) as estimated by the CDC [13] [14]. Even if hepatitis B can be better controlled in this population, the high prevalence of metabolic risk factors may negate the expected decrease in incidence of HCC in the future.

Micronesians in this study had a similar proportion of HCC detected with surveillance when compared to NMPI. When diagnosed, more Micronesians had tumor size less than $5 \mathrm{~cm}$ but the mean tumor size and proportion that met Milan criteria were similar. Some of the overall differences in tumor size between the groups may be due to the fact that more NMPI had HCC without viral risk factors or disease that warranted surveillance. Another contributing factor may be the effectiveness of surveillance in both Micronesians and NMPI where the mean BMI is high and about half of the patients are obese with BMI above 30 . Previous studies including one from this center have demonstrated that ultrasound surveillance is less reliable in obese patients [15] [16].

Multiple previous retrospective studies have demonstrated the survival benefit of HCC surveillance, which allows for detection of HCC at an earlier stage or with smaller tumor size that can be treated effectively [17] [18] [19]. Perhaps it is because $80 \%$ of Micronesians in our study had hepatitis B, a screenable disease, which allowed earlier detection and the opportunity for curative therapies and better survival. Other U.S. studies have suggested that minorities are less likely to receive transplant due to differences in education, insurance, and patient perception of involvement in their care [20] [21]. However, the Micronesians in our study had similar socioeconomic parameters and were able to overcome barriers to transplant. 
This study is limited in that it represents data from a single center with a relatively small sample size. Comparing Micronesians only to other Pacific Islanders may have limited our study's applicability, however we wanted to compare a relatively recently immigrated group to a more established population of Pacific Islanders. It is also possible that we have selected a group of inherently more motivated and financially secure Micronesians as they had the resources to make the trip from Micronesia to Hawaii. In spite of these limitations, our study describes a unique population that has not been previously characterized in the literature in terms of HCC. Because we have Hawaii's only dedicated liver center that sees approximately $70 \%$ of Hawaii's HCC cases, it is likely that we captured the majority of HCC cases in Micronesians. Finally, it is important that the U.S. is aware of this ethnic group, as Micronesians are rapidly migrating across the rest of the U.S., especially to the Midwest (Missouri, Arkansas) in search of entry-level jobs and a more affordable cost of living [22] [23]. Health issues in this ethnic group will need to be addressed.

It is remarkable that Micronesians, a new migrant population, received curative treatments on par with and had better survival than U.S.-born Pacific Islanders. Previous studies on ethnic disparities in HCC cite culturally competent education and effective communication as key factors to improve care for minorities [20]. In Hawaii, we have several health clinics that readily refer patients to our liver center and are equipped with interpreters who speak various Micronesian dialects. It is also helpful that this liver center is located on the same floor as the transplant center and the hepatobiliary/transplant surgeons, which likely facilitated navigation of the healthcare system for this population. We also use Micronesian former patients to guide new patients through the process, providing translation, cultural support and mentorship.

\section{Conclusion}

Compared to Non-Micronesian Pacific Islanders, Micronesians were as likely to have HCC discovered with screening, undergo transplant and had better survival. This study highlights the need for hepatitis B screening and HCC surveillance in Micronesians and it further demonstrates how a group of immigrants were able to overcome barriers, undergo treatment and have outcomes comparable to U.S.-born Pacific Islanders. We will need to continue efforts to monitor this group of immigrants with a high burden of liver disease. Perhaps other centers in the U.S. can develop similar strategies for other ethnic minorities and determine if this can reduce disparities in access to cancer care.

\section{Conflict of Interest}

The authors have no conflict of interest to report.

\section{Financial Support and Sponsorship}

This work was supported by U.S. National Institutes of Health grant 3P30CA071789-12S6. 


\section{References}

[1] Howlader, N., Noone, A.M., Krapcho, M., et al. (2017) SEER Cancer Statistics Review, 1975-2014, National Cancer Institute. Bethesda, MD, Based on November 2016 SEER Data Submission, posted to the SEER web site. https://seer.cancer.gov/archive/csr/1975_2013/

[2] Davila, J.A., Morgan, R.O., Shaib, Y., McGlynn, K.A. and El-Serag, H.B. (2004) Hepatitis C Infection and the Increasing Incidence of Hepatocellular Carcinoma: A Population-Based Study. Gastroenterology, 127, 1372-1380.

https://doi.org/10.1053/j.gastro.2004.07.020

[3] United States Government Accountability Office (2011) Report to Congressional Requesters: Compacts of Free Association: Improvements Needed to Assess and Address Growing Migration. GAO-12-64.

[4] U.S. Census Bureau (2012) 2010 Census Summary File 2, Compilation and Calculations by the Hawaii State Department of Business, Economic Development \& Tourism: Selected Micronesian Race Alone Group Profiles. http://files.hawaii.gov/dbedt/census/Census_2010/SF2/Selected_Micronesian_DP1_ SF2_final.pdf

[5] State of Hawaii Department of the Attorney General (2009) Final Report of the Compacts of Free Association Task Force. S.R. No. 142, S.D. 1.

[6] Hezel, F. and Levin, M. (2012) Survey of Federated States of Micronesia Migrants in the United States including Guam and the Commonwealth of Northern Mariana Islands (CNMI). FSM Office of Statistics, Budget \& Economic Management, Overseas Development Assistance and Compact Management.

https://prd.psc.isr.umich.edu/files/Resources_Report\%20on\%20PI\%20Jurisdictions. pdf

[7] Yamada, S. and Pobutsky, A. (2009) Micronesian Migrant Health Issues in Hawaii: Part 1: Background, Home Island Data, and Clinical Evidence. Californian Journal of Health Promotion, 7, 16-31.

[8] Palafox, N.A., Yamada, S., Ou, A.C., Minami, J.S., Johnson, D.B. and Katz, A.R. (2004) Cancer in Micronesia. Pacific Health Dialog, 11, 78-83.

[9] Mahoney, F.J., Woodruff, B., Auerbach, S., et al. (1996) Progress on the Elimination of Hepatitis B Virus Transmission in Micronesia and American Samoa. Pacific Health Dialog, 3, 140-146.

[10] Kowdley, K.V., Wang, C.C., Welch, S., Roberts, H. and Brosgart, C.L. (2012) Prevalence of Chronic Hepatitis B among Foreign-Born Persons Living in the United States by Country of Origin. Hepatology, 56, 422-433. https://doi.org/10.1002/hep.24804

[11] Ryerson, A.B., Eheman, C.R., Altekruse, S.F., et al. (2016) Annual Report to the Nation on the Status of Cancer, 1975-2012, Featuring the Increasing Incidence of Liver Cancer. Cancer, 122, 1312-1337.

[12] Centers for Disease Control and Prevention (2017) Adult Obesity Facts. http://www.cdc.gov/obesity/data/adult.html

[13] Galinsky, A.M., Zelaya, C.E., Simile, C. and Barnes, P.M. (2017) Health Conditions and Behaviors of Native Hawaiian and Pacific Islander Persons in the United States. National Center for Health Statistics. Vital and Health Statistics Series, 3, No. 40.

[14] Centers for Disease Control and Prevention (2017) National Diabetes Statistics Report, 2017. Centers for Disease Control and Prevention, US Department of Health and Human Services, Atlanta, GA. 
[15] Wong, L.L., Reyes, R.J., Kwee, S.A., Hernandez, B.Y., Kalathil, S.C. and Tsai, N.C. (2017) Pitfalls in Surveillance for Hepatocellular Carcinoma: How Successful is it in the Real World? Clinical and Molecular Hepatology, 23, 239-248. https://doi.org/10.3350/cmh.2017.0008

[16] Del Poggio, P., Olmi, S., Ciccarese, F., et al. (2014) Factors That Affect Efficacy of Ultrasound Surveillance for Early Stage Hepatocellular Carcinoma in Patients with Cirrhosis. Clinical Gastroenterology and Hepatology, 12, 1927. https://doi.org/10.1016/j.cgh.2014.02.025

[17] Kuo, Y.H., Lu, S.N., Chen, C.L., et al. (2010) Hepatocellular Carcinoma Surveillance and Appropriate Treatment Options Improve Survival for Patients with Liver Cirrhosis. European Journal of Cancer, 46, 744-751.

https://doi.org/10.1016/j.ejca.2009.12.018

[18] Singal, A.G., Conjeevaram, H.S., Volk, M.L., et al. (2012) Effectiveness of Hepatocellular Carcinoma Surveillance in Patients with Cirrhosis. Cancer Epidemiolology, Biomarkers and Prevention, 21, 793-799. https://doi.org/10.1158/1055-9965.EPI-11-1005

[19] Park, J.W., Chen, M., Colombo, M., et al. (2015) Global Patterns of Hepatocellular Carcinoma Management from Diagnosis to Death: The BRIDGE Study. Liver International, 35, 2155-2166. https://doi.org/10.1111/liv.12818

[20] Wilder, J.M., Oloruntoba, O.O., Muir, A.J. and Moylan, C.A. (2016) Role of Patient Factors, Preferences, and Distrust in Health Care and Access to Liver Transplantation and Organ Donation. Liver Transplantation, 22, 895-905. https://doi.org/10.1002/lt.24452

[21] Li, J., Hansen, B.E., Peppelenbosch, M.P., De Man, R.A., Pan, Q. and Sprengers, D. (2017) Factors Associated with Ethnical Disparity in Overall Survival for Patients with Hepatocellular Carcinoma. Oncotarget, 8, 15193-15204. https://doi.org/10.18632/oncotarget.14771

[22] Boiko-Weyrauch, A. (2013) Far From the Pacific, Micronesians Increasingly Call Missouri Home.

https://www.pri.org/stories/2013-04-29/far-pacific-micronesians-increasingly-callmissouri-home

[23] Walden, A. (2011) Micronesians: "It's Just Better in Arkansas". http://www.hawaiifreepress.com/ArticlesMain/tabid/56/ID/4878/Micronesians-Its-j ust-better-in-Arkansas.aspx 